\title{
Near-wall liquid film outflow of water-ethanol mixture from cylindrical channel into vacuum
}

\author{
Yuri Vyazov, Pavel Votinov, and Igor Yarygin* \\ Kutateladze Institute of Thermophysics SB RAS, Rarefied Gases Laboratory, 630090, Lavrentieva \\ ave. 1, Novosibirsk, Russia
}

\begin{abstract}
The problem of joint near-wall liquid film ejection with gas flow from a cylindrical channel into vacuum is studied experimentally. A new technique for measuring thickness and velocity of the liquid film inside the channel with the help of capacity-type probes is presented. It is shown that co-current gas flow has strong impact on the near-wall film, leading to intense wave formation, detachment of droplets from the film surface, and formation of dry spots.
\end{abstract}

\section{Introduction}

Expansion of gases, liquids and gas-liquid mixes into vacuum is of great interest for a number of scientific and technological applications. Recently a lot of attention was attracted by the jet expansion of gases from nozzles of various geometry (supersonic, transonic, axisymmetric, flat, etc.). It was established that so-called underexpanded flow is formed behind exit cross-section of a nozzle under gas expansion into vacuum. This flow includes area of free supersonic expansion (the core of the jet), as well as oblique shock waves and Mach disk [1]. The flow conditions in a jet and a set of physical processes accompanying supersonic gas expansion are mainly characterized by nozzle geometry, gas composition, its initial parameters inside the nozzle (stagnation temperature and pressure) as well as conditions in the vacuum chamber. One of the special characteristics of gas jet expansion into vacuum is Prandtl-Mayer expansion fan at the exit edge of the nozzle, in which gas turns at angles over $90^{\circ}$ relative to jet axis. Such flows are called backflows, and they form a peripheral part of the jet.

However the number of studies published concerning ejection of liquids and gas-liquid flows into vacuum is very limited and most of them are devoted to space applications, such as ejection of liquids from spacecrafts during purging of propellant refueling systems, operation of orientation thrusters in which fuel film is used for cooling walls of combustion chamber and a nozzle, etc [2,3]. As saturated vapor pressure of liquids used is usually several orders of magnitude higher than pressure in surrounding space, liquid becomes "instantly overheated" being ejected into vacuum. This leads to liquid film boiling, its disintegration into droplets, and phase transitions on the surface and inside droplets. Also the co-current flow of gas has significant effect on behavior of a wall liquid film both when moving inside the channel and under ejection into vacuum. In experimental studies [4] it

${ }^{*}$ Corresponding author : yarygin@itp.nsc.ru 
was shown that at the exit edge of the nozzle the wall film of liquid not only breaks up into droplets, but also emerges on the external surface of the channel, moving on it in the opposite direction, even against gravitation force. Under ejection into atmosphere the revealed effect is absent, and the ordinary gas-droplet flow takes place behind the exit cross-section of the channel. Another important feature of wall liquid film behavior inside the channel noted in [5] is presence of high-velocity co-current gas flow. Gas velocity under expansion from the cylindrical channel into vacuum reaches sonic velocity at the exit cross-section. This leads to rather high (over 10) values of Weber number We of co-current gas flow. According to current understanding [6] detachment of droplets from the film surface and their carrying away by co-current gas flow takes place at Weber numbers $\mathrm{We}>2$. Experimental studies [5] have shown that amount of liquid carried away by gas flow can reach significant values (tens of percent of the liquid initial flow rate). Note that only ethanol was used as working liquid in studies [4,5]. Current paper is devoted to experimental study of water-ethanol mixtures (with concentration from 0 to $100 \%$ ) ejection with co-current gas flow from cylindrical channel into rarefied space. Some aspects of the problem concerning behavior of near-wall films of binary mixtures at the exit edge were presented in [7], and special attention in this paper is paid to interaction of the liquid film with co-current flow inside the channel.

\section{Experimental setup and measurement techniques}

The main goal of our experiments was to perform measurements of wall liquid film local parameters (thickness and velocity). Current methods of liquid film diagnostics can be separated into two classes: contact (probe) and non-contact (optical). In our study the probe method was chosen, namely the method using capacity-type probes [8]. The scheme of test section is shown in Fig. 1.

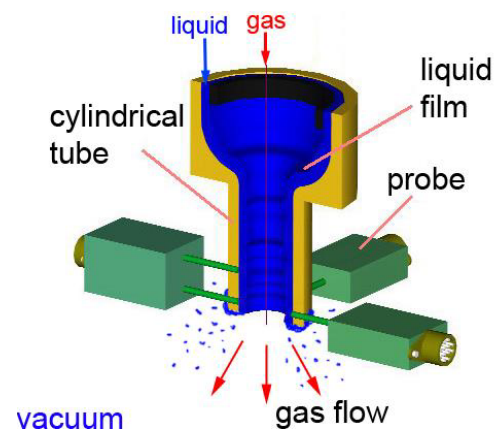

Fig. 1. Scheme of test section.

The key idea of capacity-type probe diagnostics is to measure liquid layer capacity over the probe and to convert probe indications into film thickness with the help of special calibration technique. This method has good both spatial (about $1 \mathrm{~mm}$ ) and temporal (about $1 \mathrm{~ms}$ ) resolutions. Two sequentially located probes 3 were used for wall film velocity measurement. Four probes 2 located through $90^{\circ}$ were used for thickness measurement. Experiments were carried out on the VIKING vacuum gas-dynamic setup at Kutateladze Institute of Thermophysics [9]. The large volume of the working chamber $\left(150 \mathrm{~m}^{3}\right)$ allowed operation in pulse mode with high flow rates of gas and liquid. Cylindrical channel (nozzle) made of brass with $5 \mathrm{~mm}$ diameter, $20 \mathrm{~mm}$ length and $1 \mathrm{~mm}$ wall thickness was mounted vertically in the vacuum chamber, with exit part facing downwards. The liquid entered through a circular gap of $0.1 \mathrm{~mm}$ width into the stagnation chamber of the nozzle and moved down the nozzle walls as a film. Simultaneously, gas was blown through the nozzle. 
Purified air was used as working gas, and water-ethanol mixture with volumetric concentration of 0,50 and $100 \%$ was used as working liquid. It should be mentioned that viscosity of water-ethanol mixture can exceed viscosity of pure components by several factors depending on concentration and temperature. Initially gas and liquid were at room temperature. Experiments were carried out in pulse modes. Usually the pulse length was 5 seconds, and the pressure in vacuum chamber increased from initial $1 \mathrm{~Pa}$ up to less than 1.1 $\mathrm{Pa}$. Mass flow rate of gas and liquid varied from 0.5 to $20 \mathrm{~g} / \mathrm{s}$ and from 0.6 to $2 \mathrm{~g} / \mathrm{s}$, respectively.

\section{Results}

An example of relationship between liquid film thickness and time under expansion with co-current gas flow from the cylindrical channel into vacuum is shown in Fig. 2 for ethanol (a) and water (b).
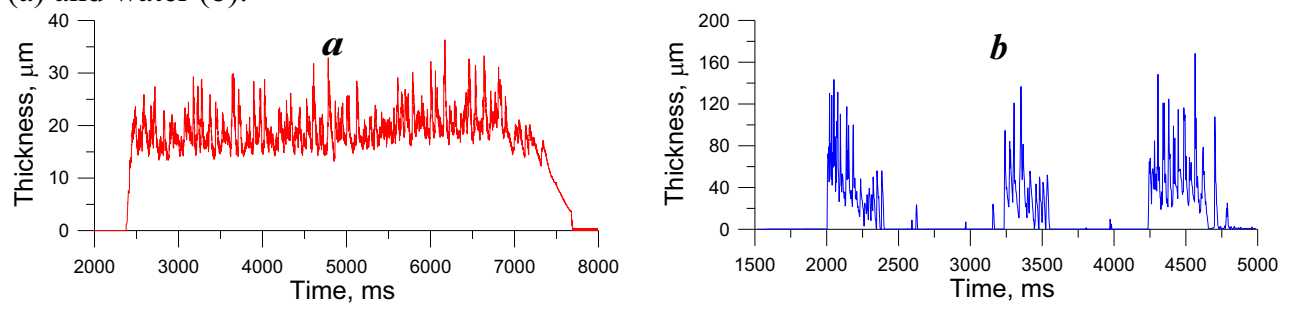

Fig. 2. Liquid film thickness. $\operatorname{Re}_{\text {gas }}=4 \cdot 7 \cdot 10^{4}$. $\mathrm{a}$ - ethanol film, $\mathrm{b}-$ water film.

From Fig. 2a one can see timing of liquid film arrival to the probe. Further liquid film with thickness of several tens of microns is registered on the probe during feeding time. This film has wave structure with rather high amplitude and frequency. Then rapid drop of film thickness happens after liquid supply is cut off. Waves on the film surface have steep front and flat rolloff. We believe that wave characteristics mainly depend on parameters of co-current gas flow (its Reynolds number). Liquid film behavior changes under ejection of water. Under certain condition we observed multiple arrivals of film to the probe and decrease of film thickness to zero. At the same time average film thickness was rather high. Such behavior of the film seems to be caused by formation of dry spots on the channel surface and film flow in the form of separate streamlets - rivulets. This is probably due to high value of water surface tension coefficient and low wettability of the surface.

Experimental data on measurements of liquid film thickness under motion with highvelocity co-current gas flow inside the channel are shown in Fig. 3 for ethanol, water and their mixture.

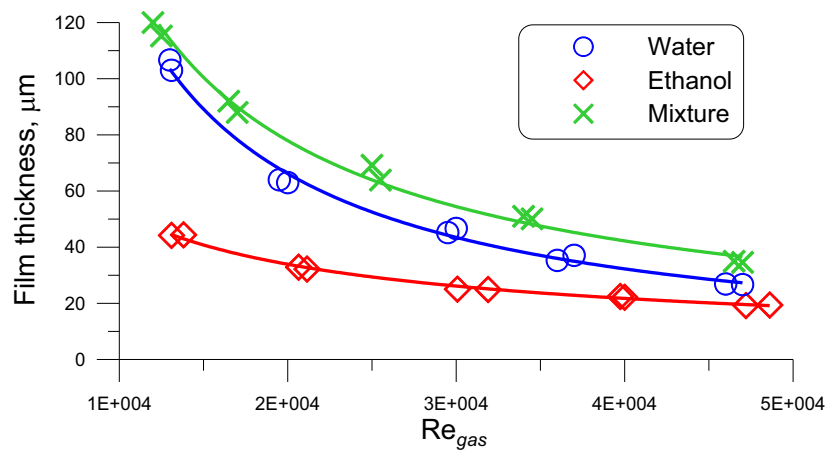

Fig. 3. Dependence of liquid film thickness on Reynolds number of gas flow. 
One might expect that the film thickness for water, which has the lowest viscosity among working liquids, should be the lowest among all the working liquids used at identical Reynolds numbers of the film. At the same time for water-ethanol mixture with concentration of $50 \%$ the thickness should be the highest. Film thickness of mixture was indeed the highest, however film thickness for water was much higher, than that for ethanol. It is caused by formation of dry spots and the rivulet flow mode of water film. Note presence of droplets detachment from liquid film surface in our experiments that depends on Weber number of co-current gas flow. Since Weber number is in inverse proportion to coefficient of surface tension of liquid, the highest Weber numbers are achieved for film flows of ethanol and water-ethanol mixture. Under conditions of our experiments Weber numbers exceeded 100 that lead to very intensive (tens of percent from the initial flow rate of liquid) detachment of droplets from the film surface along the channel and decrease of film thickness at the channel exit cross-section.

\section{Conclusion}

A series of experimental studies on liquid film interaction with high-velocity gas flow under expansion from a cylindrical channel into vacuum was carried out. These experiments allowed measuring average thickness and velocity of near-wall liquid film, and establishing special features of film interaction with gas flow (wave formation, detachment of droplets from film surface by gas flow). It was found that under expansion into the vacuum physical properties of liquids and parameters of co-current gas flow determine film behavior inside the channel. It was shown that surface tension coefficient of liquid essentially affect rupture of thin films, formation of dry spots on the channel surface and transition to a rivulet flow mode. Our results expand existing knowledge on the features of liquids ejection from channels into the vacuum in the form of thin films, and could be relevant for verifying two-phase flow models.

The study was supported by the Russian Foundation for Basic Research, research project No. 16-3800406 .

\section{References}

1. S. Crist, P. Sherman, D. Glass, AIAA J. 4, 68 (1966)

2. H. Trinks, I. Kaelsch, AIAA Paper No. 87-1603 (1987)

3. V. Yarygin, Yu. Gerasimov, A. Krylov, V. Prikhodko, I. Yarygin, Microgravity Sci. Technol. 23, 15 (2011)

4. V.G. Prikhodko, S.F. Chekmarev, V.N. Yarygin, I.V. Yarygin, Doklady Phys. 49, 119 (2004)

5. V. Prikhodko, I. Yarygin and V. Yarygin, Microgravity Sci. Technol. 21, 277 (2009)

6. I.I. Gogonin, J. Eng. Phys. Thermoph. 77, 454 (2004)

7. Yu. Vyazov, V. Prikhodko, I. Yarygin, V. Yarygin, MATEC Web of Conferences 84, 00040 (2016)

8. A.F. Serov, S.V. Kotov, A.D. Nazarov, A.N. Pavlenko, N.I. Pecherkin, V.Yu. Chehovich, Instrum. Exp. Tech. 40, 136 (1997)

9. V.G. Prikhodko, G.A. Khramov, V.N. Yarygin, Instrum. Exp. Tech. 39, 309 (1996) 